\title{
A Powerful and Robust Test in Genetic Association Studies
}

\author{
Kuang-Fu Cheng Jen-Yu Lee \\ Biostatistics Center and School of Public Health, Taipei Medical University, Taipei, Taiwan, ROC
}

\section{Key Words}

Association test · Genetic model · Power · Robustness · SNP

\begin{abstract}
There are several well-known single SNP tests presented in the literature for detecting gene-disease association signals. Having in place an efficient and robust testing process across all genetic models would allow a more comprehensive approach to analysis. Although some studies have shown that it is possible to construct such a test when the variants are common and the genetic model satisfies certain conditions, the model conditions are too restrictive and in general difficult to verify. In this paper, we propose a powerful and robust test without assuming any model restrictions. Our test is based on the selected $2 \times 2$ tables derived from the usual $2 \times 3$ table. By signals from these tables, we show through simulations across a wide range of allele frequencies and genetic models that this approach may produce a test which is almost uniformly most powerful in the analysis of low- and high-frequency variants. Two cancer studies are used to demonstrate applications of the proposed test.
\end{abstract}

\section{KARGER}

(c) 2014 S. Karger AG, Basel

0001-5652/14/0781-0038\$39.50/0

E-Mail karger@karger.com

www.karger.com/hhe

\section{Introduction}

In genetic epidemiology, genotypic data of single nucleotide polymorphisms (SNPs) from diseased and nondiseased individuals are compared to determine if there exists a gene-disease association. However, to optimally evaluate a gene-disease association signal, one needs to assume the correct inheritance model, so that the power of the test can be maximized. Many test statistics are used in association studies. The most popular test among them is the Cochran-Armitage trend test $\left(\mathrm{CATT}_{\mathrm{ADD}}\right)$ based on an additive (ADD) genetic model. Different CATTs are also used if the underlying genetic model is known to be recessive (REC), dominant (DOM) or multiplicative (MUL) [1-3]. Unfortunately, these tests are often underpowered if the genetic model is incorrectly specified.

If the genetic model is unknown, other test statistics often used are Pearson's 2-df $\chi^{2}$ (independence) test statistic $\left(\chi_{2}^{2}\right)$ or the max-statistic (MAX3), which is the largest absolute value of CATTs across the REC, DOM and ADD models $[4,5]$. The Wellcome Trust Case-Control Consortium [6] considered the minimum $p$ values of $\mathrm{CATT}_{\mathrm{ADD}}$ and $\chi_{2}^{2}$. This test is called MIN2. Joo et al. [7] derived the asymptotic null distribution of MIN2 and

Kuang-Fu Cheng

Biostatistics Center and School of Public Health, Taipei Medical University $250 \mathrm{Wu}$-Shing Street

Taipei 110, Taiwan (ROC)

E-Mail kfcheng@tmu.edu.tw 
compared it with other competing tests by simulations. Another max-type test statistic is CLRT $=\max \left(C_{\text {CLRT }}\right.$ REC, $\left.C_{\text {CLRT }}{ }_{\text {DOM }}\right)$, where CLRT $_{\text {REC }}\left(\mathrm{CLRT}_{\mathrm{DOM}}\right)$ is the constrained likelihood ratio test when the underlying genetic model is restricted to the REC (DOM) model. The asymptotic distributions of CLRT and MAX3 and their approximations have been studied by Wang and Sheffield [8], Conneely and Boehnke [9] and Li et al. [10].

Consider a SNP with alleles $A$ and a with frequencies $P$ and $1-P=Q$, respectively. We assume that $\mathrm{A}$ is the less common (minor) allele. The genotype variable is defined by $G=j$, if the subject carries $j$ copies of $A, j=0,1,2$. Let $R=\operatorname{Pr}$ (disease) be the disease prevalence and $f_{j}=\operatorname{Pr}$ (disease $\mid G=j$ ) the penetrance of the genotype $G=j$. The genotype-relative risks (GRRs) are defined by $\lambda_{j}=f_{j} / f_{0}$. The REC, DOM, ADD and MUL models refer to $\lambda_{1}=1$, $\lambda_{2},\left(1+\lambda_{2}\right) / 2$ and $\lambda_{2}^{1 / 2}$, respectively. They belong to the model space $M\left(=\left\{1 \leq \lambda_{1} \leq \lambda_{2}\right\} \cup\left\{1=\lambda_{1}<\lambda_{2}\right\}\right) \cup M^{*}(=$ $\left.\left\{\left(\lambda_{1}, \lambda_{2}\right): 1>\lambda_{1} \geq \lambda_{2}>0\right\} \cup\left\{1=\lambda_{1}>\lambda_{2}>0\right\}\right)$, where one of the alleles has a higher risk. The CATTs are modelbased tests and they have been shown to be more powerful when the genetic model is known, but they could be underpowered when the genetic model is incorrect. From their simulation results, Joo et al. [7] found that for SNPs with a minor allele frequency (MAF) $>0.10$, MAX3 and CLRT tended to have a better power performance if the genetic models were restricted to the REC, DOM, ADD or MUL models. On the other hand, Pearson's $\chi_{2}^{2}$ and MIN2 were more efficient if the genetic model space contained the overdominant and underdominant models $\left(f_{1}>f_{0}, f_{1}>f_{2}\right.$ or $\left.f_{1}<f_{0}, f_{1}<f_{2}\right)$.

Joo et al. [7] concluded that there was no single best test for detecting SNP-disease association signals. They suggested that efficiency-robust tests, depending on specific assumption of genetic models, are preferable. Unfortunately, one is often unable to verify the validity of the model assumption if it is too restrictive. On the other hand, if the model assumption is too general, it becomes difficult to conclude which test should be used in the association studies. Recently, Chen and Ng [11] have considered the assumption that the genetic models belong to the model space $M \cup M^{*}$; i.e. the SNP's GRRs are monotonically increasing or decreasing. Based on their simulations, they showed that, compared with other tests, their test usually had the highest or second-highest power when the genetic model was not REC, DOM, ADD or MUL. However, the power gain of the new test was not substantial. Further, the model assumption is still too restrictive, since so far there is no method available for testing the validity of the monotonicity assumption.
In this paper, we propose a simple method for constructing a generally more powerful association test without requiring any model condition. Thus our test can be applied simultaneously in the model space that contains overdominant (underdominant) models along with genetic models with monotonic relative risks. Since there has been increasing interest in analyzing next-generation sequencing data $[12,13]$, we also study whether this method can be applied to study associations between complex traits and SNPs with a low MAF $(0.01 \leq \mathrm{MAF} \leq$ 0.05 ) or a high MAF (MAF $>0.05$ ) and a deleterious or protective effect. Our simulation results confirmed that the proposed test is almost uniformly most powerful. Compared with other well-known tests, the power gain of the new test is also quite substantial in some situations.

In the Methods section, we give a formal definition for the new association test. Then we present the simulation results including applications to an ovarian cancer study and a prostate cancer study. In the Discussion section, we also point out a possible generalization of our method to simultaneously detect the main and interaction (SNP-environment or SNP-SNP) effects of a SNP. Using the same principle, our method also can be applied to detect an association signal from a group of rare variants $(\mathrm{MAF}<1 \%)$ in a gene region $[14,15]$.

\section{Methods}

\section{Notations}

We denote the genotype frequency of a SNP as $g_{j}=\operatorname{Pr}(G=j)$. Then, $g_{0}=Q^{2}+F P Q, g_{1}=2(1-F) P Q$ and $g_{2}=P^{2}+F P Q$, where $F$ is Wright's coefficient of inbreeding. If the Hardy-Weinberg equilibrium condition holds, then $F=0$. Let $D=1$ denote that the subject is diseased and $D=0$ otherwise. The genotype data can be summarized in a $D \times G$ table. From this $2 \times 3$ table, one can create multiple $2 \times 2$ tables for detecting an association signal. We first defined 12 binary variables $G_{k}$ and $G_{k}^{*}, k=1, \ldots, 6$. The definitions of $G_{k}$ are given in table 1 and $G_{k}^{*}=1-G_{k}$. Next, we propose using 12 tables $T_{k}=D \times G_{k}$ and $T_{k}^{*}=D \times G_{k}^{*}, k=1, \ldots, 6$, to construct test statistics. Our idea is that if the $D \times G$ table carries some association signal, then there must be at least one $2 \times 2$ table $T_{k}$ or $T_{k}^{*}$ carrying a similar signal. On the other hand, if there is no association, then no signal can be detected from these tables either.

\section{A Robust Test Based on the Selected $2 \times 2$ Tables}

Suppose we have six $2 \times 2$ tables $T_{k}$ as described above. Assume that in the $k$-th $(k=1, \ldots, 6)$ table we have observed $N_{1+, k}$ subjects from the case population and among them there are $X_{10, k}$ subjects taking $G_{k}=0$. Let the corresponding numbers observed from the control population be denoted by $N_{0+, k}$ and $X_{00, k}$. Thus the total number with $G_{k}=0$ in the cases and controls is $N_{+0, k}=X_{00, \mathrm{k}}+$ $\mathrm{X}_{10, k}$ and the total number with $G_{k}=1$ is $N_{+0, k}=X_{01, k}+X_{11, k}$. Based on this setup, there are several quantities that can be used 
Table 1. The definitions of $G_{k}$

\begin{tabular}{lllllll}
\hline$G_{k}=$ & $k=$ & & & \\
\cline { 2 - 6 } & 1 & 2 & 3 & 4 & 5 & 6 \\
\hline 0 & $G=0$ & $G=0$ & $G=0$ & $G=0$ or 1 & $G=0$ or 2 & $G=1$ \\
1 & $G=1$ & $G=2$ & $G=1$ or 2 & $G=2$ & $G=1$ & $G=2$ \\
\hline
\end{tabular}

Table 2. Empirical type I error rates of the competing tests

\begin{tabular}{llllll}
\hline Sample size & MAF & MAX3 & CLRT & MIN2 & IND $^{\mathrm{a}}$ \\
\hline 2,000 & 0.01 & 0.038 & 0.027 & 0.025 & $0.038(0.034)$ \\
1,500 & 0.05 & 0.040 & 0.028 & 0.035 & $0.045(0.043)$ \\
1,000 & 0.1 & 0.046 & 0.034 & 0.048 & $0.048(0.047)$ \\
500 & 0.2 & 0.043 & 0.033 & 0.049 & $0.048(0.046)$ \\
\hline
\end{tabular}

Sample size $N=N_{0}=N_{1}$.

a The values in parentheses are computed based on using the asymptotic distribution.

to measure the $D-G_{k}$ association signal. Here, we consider the simple statistic defined by $\left(N_{k} X_{10, k}-\mathrm{N}_{1+, \mathrm{k}} N_{+0, k}\right)^{2}$, where $N_{k}=N_{0+, k}+$ $N_{1+, k}$. Note that this simple statistic is invariant with respect to the row or column permutations in the $2 \times 2$ table, and hence tables $T_{k}$ and $T_{k}^{*}$ give the same statistic. This means that the statistic can be used to detect an association signal for deleterious or protective SNPs.

The standardized statistic is given by

$$
S_{k}^{2}=N_{k}\left(N_{k} X_{10, k}-N_{1+, k} N_{+0, k}\right)^{2} /\left(N_{1+, k} N_{+0, k} N_{0+, \mathrm{k}} N_{1+, \mathrm{k}}\right),
$$

which follows a $1-\mathrm{df} \chi^{2}$ distribution when there is no association signal. Most $S_{k}^{2}$ tests based on using different $T_{k}$ tables are powerful in detecting an association signal under certain genetic models, but the tables may be largely noise under other models. For example, our simulations showed that $S_{4}^{2}$ is only powerful under the REC genetic model, but it is generally very poor in other genetic models. Also, $S_{1}^{2}$ and $S_{5}^{2}\left(S_{2}^{2}\right.$ and $\left.S_{6}^{2}\right)$ showed a similar power performance under the REC model, but $S_{1}^{2}\left(S_{2}^{2}\right)$ generally dominated over $S_{5}^{2}\left(S_{6}^{2}\right)$ in power under other genetic models. Thus, after excluding the inferior tables, we propose combining information from the first 3 tables to formally define an independence test with statistic:

$$
\mathrm{IND}=\sum_{k=1}^{3} S_{k}^{2}
$$

This test is similar in form to the sum of squared score test [16] and to the sequence kernel association test [17]. They only differ in using the weighting methods. The $\mathrm{p}$ value of the IND test can be approximated by using the usual permutation argument [or bootstrap method 18]. Another approach is to use the asymptotic distribution. Note that asymptotically, IND is equivalent to a weighted sum of 3 correlated $\chi^{2}$ random variables (see Appendix). Thus, the $\mathrm{p}$ value can also be approximated by comparing the observed IND with the INDs simulated from a multivariate normal distribution. The computation time can be reduced substantially if

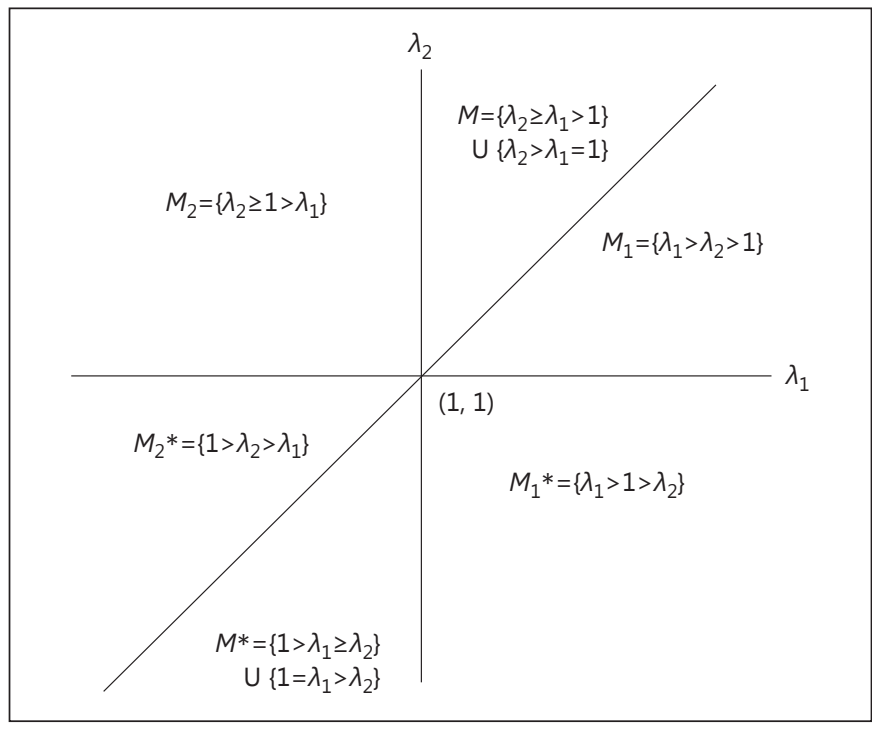

Fig. 1. Genetic models defined by their GRRs.

the asymptotic-based IND test is used instead of the permutationbased IND. Free software for computing the IND test's p value is available at http://biostat.tmu.edu.tw/index.php/10-all/20-download-chengkf.

\section{Simulations}

We used simulations to study the properties of the 4 competing tests: MAX3, CLRT, MIN2 and IND. Given a MAF $p$, we defined the genotype frequencies $g_{j}$ under the Hardy-Weinberg equilibrium condition. We assumed a disease prevalence $R=0.01$ across all simulations. Thus if the GRRs $\lambda_{1}$ and $\lambda_{2}$ are given, then the penetrance of the genotype $G=j$ is given by $f_{j}=f_{0} \lambda_{j}, j=0,1,2$, where $f_{0}=R /\left(g_{0}+g_{1} \lambda_{1}+g_{2} \lambda_{2}\right)$. This implies that the genotype $G=j$ has frequency $p_{j}=f_{j} g_{j} / R$ in the case population and frequency $q_{1}=$ $\left(1-f_{j}\right) g_{j} /(1-R)$ in the control population. In the simulations, the case and control data were sampled from a multinomial distribution with probabilities $\left(p_{0}, p_{1}, p_{2}\right)$ and $\left(q_{0}, q_{1}, q_{2}\right)$ with sample size $N_{1}$ and $N_{0}$, respectively.

The MAFs used in all simulations were $0.20,0.10,0.05$ and 0.01 . The following case and control sample sizes were used: $N=N_{1}=$ $N_{0}=500,1,000,1,500$ or 2,000 , in order to maintain appropriate power for comparison. The GRRs $\lambda_{1}, \lambda_{2}=1.2$ or 1.5 and $1.2^{-1}$ or $1.5^{-1}$ were used in the power simulations. Six different genetic model spaces were considered for power comparisons; they are defined in figure 1 . Space $M_{1}$ contains the overdominant models, for example. We also studied the efficiency robustness of all competing tests. Here we compared the minimum powers of the competing tests across all different genetic models. The GRRs were selected so that the power of the best test was about $80 \%$ [see also Joo et al. 7].

In all simulations, the significance level was taken to be 0.05 . Under each simulation condition, empirical type I error rates and power were estimated based on 5,000 simulation replications. The asymptotic results were used to compute the $\mathrm{p}$ values for the CLRT and 
Table 3. Empirical power of the competing tests under the 4 common genetic models and when the SNPs have positive log GRRs

\begin{tabular}{lrrrrrrr}
\hline Model & $\lambda_{2}$ & $\begin{array}{l}\text { Sample } \\
\text { size }\end{array}$ & MAF & MAX3 & CLRT & MIN2 & IND \\
\hline DOM & 1.5 & 2,000 & 0.01 & 0.564 & 0.505 & 0.498 & 0.564 \\
& & 1,500 & 0.05 & 0.968 & 0.935 & 0.966 & 0.975 \\
& & 1,000 & 0.10 & 0.980 & 0.964 & 0.983 & 0.988 \\
& & 500 & 0.20 & 0.908 & 0.839 & 0.910 & 0.937 \\
\hline ADD & 1.5 & 2,000 & 0.01 & 0.183 & 0.143 & 0.139 & 0.185 \\
& & 1,500 & 0.05 & 0.528 & 0.409 & 0.531 & 0.562 \\
& & 1,000 & 0.10 & 0.606 & 0.520 & 0.637 & 0.656 \\
& & 500 & 0.20 & 0.519 & 0.410 & 0.543 & 0.565 \\
\hline MUL & 1.5 & 2,000 & 0.01 & 0.160 & 0.124 & 0.117 & 0.159 \\
& & 1,500 & 0.05 & 0.445 & 0.337 & 0.445 & 0.484 \\
& & 1,000 & 0.10 & 0.531 & 0.445 & 0.561 & 0.575 \\
& 500 & 0.20 & 0.489 & 0.367 & 0.510 & 0.523 \\
\hline REC & 1.5 & 2,000 & 0.01 & 0.039 & 0.028 & 0.024 & 0.039 \\
& & 1,500 & 0.05 & 0.056 & 0.047 & 0.057 & 0.058 \\
& 1,000 & 0.10 & 0.138 & 0.124 & 0.135 & 0.107 \\
& 500 & 0.20 & 0.241 & 0.206 & 0.232 & 0.165 \\
\hline
\end{tabular}

Sample size $N=N_{0}=N_{1}$.
Table 4. Empirical power of the competing tests under the 4 common genetic models and when the SNPs have negative log GRRs

\begin{tabular}{lrrrrrrr}
\hline Model & $\lambda_{2}$ & $\begin{array}{c}\text { Sample } \\
\text { size }\end{array}$ & MAF & MAX3 & CLRT & MIN2 & IND \\
\hline DOM & $1.5^{-1}$ & 2,000 & 0.01 & 0.378 & 0.325 & 0.308 & 0.380 \\
& & 1,500 & 0.05 & 0.902 & 0.826 & 0.885 & 0.911 \\
& & 1,000 & 0.10 & 0.926 & 0.878 & 0.931 & 0.947 \\
& & 500 & 0.20 & 0.862 & 0.798 & 0.869 & 0.901 \\
\hline ADD & $1.5^{-1}$ & 2,000 & 0.01 & 0.104 & 0.081 & 0.074 & 0.105 \\
& & 1,500 & 0.05 & 0.342 & 0.240 & 0.307 & 0.350 \\
& & 1,000 & 0.10 & 0.393 & 0.311 & 0.419 & 0.434 \\
& & 500 & 0.20 & 0.391 & 0.296 & 0.409 & 0.423 \\
\hline MUL & $1.5^{-1}$ & 2,000 & 0.01 & 0.128 & 0.103 & 0.092 & 0.128 \\
& & 1,500 & 0.05 & 0.396 & 0.295 & 0.367 & 0.410 \\
& & 1,000 & 0.10 & 0.452 & 0.356 & 0.478 & 0.496 \\
& 500 & 0.20 & 0.433 & 0.341 & 0.457 & 0.472 \\
\hline REC & $1.5^{-1}$ & 2,000 & 0.01 & 0.042 & 0.031 & 0.027 & 0.043 \\
& & 1,500 & 0.05 & 0.050 & 0.035 & 0.044 & 0.052 \\
& 1,000 & 0.10 & 0.083 & 0.076 & 0.085 & 0.069 \\
& 500 & 0.20 & 0.169 & 0.147 & 0.166 & 0.117 \\
\hline
\end{tabular}

Sample size $N=N_{0}=N_{1}$.

ic-based IND test also performed well in terms of type I errors, except in the case of very small SNP frequencies. In the latter case, however, the type I error rates of the permutation-based and asymptotic-based IND tests were both underestimated and their difference was very small.

The power results are given in tables 3-5. In table 3, the power results are shown when the genetic model was DOM, ADD, MUL or REC and the log GRRs were positive and, in table 4 , when they were negative. Table 5 shows the power results when other genetic models, including over- and underdominant models, were assumed. First, the permutation-based IND test was uniformly most powerful among the 4 competing tests, irrespective of whether the results were obtained under the DOM, ADD, MUL and REC model (as given in tables 3 and 4) or under other arbitrary genetic models (as given in table 5) and independent of whether high- or low-frequency SNPs with positive, negative or mixed log GRRs were used. The second-best test was MAX3 in the case of lowfrequency SNPs. In the case of high-frequency SNPs, the second-best test was MIN2. This is in agreement with the results reported by Joo et al. [7] in their analysis of common variants. According to the simulation results shown in table 5, if one uses the permutation-based IND test instead of the MIN2 test, the largest power improvement is 
Table 5. Empirical power of the competing tests under arbitrary genetic models and when the SNPs have different $\log$ GRR directions

\begin{tabular}{|c|c|c|c|c|c|c|c|}
\hline Model & $\lambda_{1}, \lambda_{2}$ & Sample size & MAF & MAX3 & CLRT & MIN2 & $\mathrm{IND}^{\mathrm{a}}$ \\
\hline$M$ & $1.2,1.5$ & $\begin{array}{r}2,000 \\
1,500 \\
1,000 \\
500\end{array}$ & $\begin{array}{l}0.01 \\
0.05 \\
0.10 \\
0.20\end{array}$ & $\begin{array}{l}0.133 \\
0.383 \\
0.470 \\
0.431\end{array}$ & $\begin{array}{l}0.101 \\
0.283 \\
0.392 \\
0.339\end{array}$ & $\begin{array}{l}0.094 \\
0.385 \\
0.498 \\
0.457\end{array}$ & $\begin{array}{l}0.133(0.126) \\
0.412(0.406) \\
0.511(0.502) \\
0.463(0.469)\end{array}$ \\
\hline$M^{*}$ & $1.2^{-1}, 1.5^{-1}$ & $\begin{array}{r}2,000 \\
1,500 \\
1,000 \\
500\end{array}$ & $\begin{array}{l}0.01 \\
0.05 \\
0.10 \\
0.20\end{array}$ & $\begin{array}{l}0.117 \\
0.341 \\
0.392 \\
0.388\end{array}$ & $\begin{array}{l}0.091 \\
0.253 \\
0.304 \\
0.305\end{array}$ & $\begin{array}{l}0.083 \\
0.315 \\
0.420 \\
0.415\end{array}$ & $\begin{array}{l}0.117(0.109) \\
0.358(0.343) \\
0.431(0.431) \\
0.423(0.420)\end{array}$ \\
\hline$M_{1}$ & $1.5,1.2$ & $\begin{array}{r}2,000 \\
1,500 \\
1,000 \\
500\end{array}$ & $\begin{array}{l}0.01 \\
0.05 \\
0.10 \\
0.20\end{array}$ & $\begin{array}{l}0.570 \\
0.971 \\
0.965 \\
0.850\end{array}$ & $\begin{array}{l}0.509 \\
0.938 \\
0.944 \\
0.810\end{array}$ & $\begin{array}{l}0.504 \\
0.968 \\
0.971 \\
0.860\end{array}$ & $\begin{array}{l}0.571(0.545) \\
0.977(0.970) \\
0.979(0.983) \\
0.901(0.902)\end{array}$ \\
\hline$M_{1}^{*}$ & $1.5,1.2^{-1}$ & $\begin{array}{r}2,000 \\
1,500 \\
1,000 \\
500\end{array}$ & $\begin{array}{l}0.01 \\
0.05 \\
0.10 \\
0.20\end{array}$ & $\begin{array}{l}0.551 \\
0.961 \\
0.956 \\
0.819\end{array}$ & $\begin{array}{l}0.489 \\
0.941 \\
0.952 \\
0.861\end{array}$ & $\begin{array}{l}0.482 \\
0.958 \\
0.969 \\
0.885\end{array}$ & $\begin{array}{l}0.554(0.541) \\
0.972(0.969) \\
0.980(0.981) \\
0.898(0.899)\end{array}$ \\
\hline$\overline{M_{2}}$ & $1.5^{-1}, 1.2$ & $\begin{array}{r}2,000 \\
1,500 \\
1,000 \\
500\end{array}$ & $\begin{array}{l}0.01 \\
0.05 \\
0.10 \\
0.20\end{array}$ & $\begin{array}{l}0.380 \\
0.861 \\
0.862 \\
0.697\end{array}$ & $\begin{array}{l}0.331 \\
0.825 \\
0.874 \\
0.806\end{array}$ & $\begin{array}{l}0.308 \\
0.868 \\
0.901 \\
0.822\end{array}$ & $\begin{array}{l}0.385(0.376) \\
0.902(0.894) \\
0.927(0.928) \\
0.829(0.833)\end{array}$ \\
\hline$M_{2}{ }^{*}$ & $1.5^{-1}, 1.2^{-1}$ & $\begin{array}{r}2,000 \\
1,500 \\
1,000 \\
500 \\
\end{array}$ & $\begin{array}{l}0.01 \\
0.05 \\
0.10 \\
0.20 \\
\end{array}$ & $\begin{array}{l}0.380 \\
0.882 \\
0.904 \\
0.799 \\
\end{array}$ & $\begin{array}{l}0.326 \\
0.819 \\
0.861 \\
0.757 \\
\end{array}$ & $\begin{array}{l}0.311 \\
0.867 \\
0.915 \\
0.815 \\
\end{array}$ & $\begin{array}{l}0.383(0.345) \\
0.897(0.897) \\
0.935(0.937) \\
0.857(0.857)\end{array}$ \\
\hline
\end{tabular}

$41.5 \%$, which occurs in the case of model $M$ and with a $\mathrm{MAF}=0.01$. The corresponding largest power improvements are 18.9 and $45.6 \%$, when one uses the permutation-based IND test instead of the MAX3 and CLRT tests, respectively. The former occurs in the case of model $M_{2}{ }^{*}$ and with a MAF $=0.2$ and the latter in the case of model $M$ and with a MAF $=0.05$. Under a REC model, all tests have very low power as expected, and it seems meaningless to discuss which test is more powerful. Even with an additional 1,000 observations in each case and control sample, the power improvement was marginal (results not shown). This is particularly true when the SNP has a low frequency. Under the REC model, however, MAX3 and MIN2 had better power performances. Table 5 also shows that for the high-frequency SNPs, the power performance of the permutation-based or asymptotic-based IND test was very similar. For the low-frequency SNPs, the asymptotic-based IND test was slightly underpow- ered. This is because the empirical type I error rate of the asymptotic-based IND test was smaller than that of the permutation-based IND test. However, their power differences were very small, in general.

According to Joo et al. [7], if the true genetic model is totally unknown, a useful measure to compare different tests is the efficiency robustness $[4,19]$. Table 6 gives some simulated results about the efficiency robustness of the 4 competing tests under different genetic models and with different MAFs. In table 6, we can observe, first of all, that the minimum power of MAX3 with the 4 different MAFs ranges from 0.656 to 0.795 . The corresponding ranges for CLRT, MIN2 and the permutation-based IND tests are $0.660-0.746,0.730-0.755$ and $0.794-0.797$, respectively. We find that the permutation-based IND test has the largest minimum power across all simulated conditions. Thus, it has the greatest efficiency robustness. In the case of low-frequency SNPs, MAX3 has a minimum 
Table 6. Efficiency robustness of the competing tests under various genetic models

\begin{tabular}{|c|c|c|c|c|c|c|c|c|}
\hline $\begin{array}{l}\text { Sample } \\
\text { size }\end{array}$ & MAF & Model & $\lambda_{1}$ & $\lambda_{2}$ & MAX3 & CLRT & MIN2 & IND \\
\hline \multirow{6}{*}{2,000} & \multirow[t]{6}{*}{0.01} & $M$ & 1.68 & 1.88 & 0.805 & 0.752 & 0.760 & 0.805 \\
\hline & & $M^{*}$ & $2.04^{-1}$ & $2.35^{-1}$ & 0.802 & 0.761 & 0.738 & 0.803 \\
\hline & & $M_{1}$ & 1.68 & 1.40 & 0.808 & 0.754 & 0.754 & 0.809 \\
\hline & & $M_{1}^{*}$ & 1.67 & $1.42^{-1}$ & $\underline{0.795}$ & $\underline{0.746}$ & 0.737 & $\underline{0.797}$ \\
\hline & & $M_{2}$ & $2.05^{-1}$ & 1.70 & 0.798 & 0.765 & $\underline{0.730}$ & 0.806 \\
\hline & & $M_{2}^{*}$ & $2.05^{-1}$ & $1.70^{-1}$ & 0.800 & 0.766 & 0.745 & 0.801 \\
\hline \multirow[t]{6}{*}{1,500} & \multirow[t]{6}{*}{0.05} & $M$ & 1.34 & 1.63 & 0.768 & 0.677 & 0.774 & 0.797 \\
\hline & & $M^{*}$ & $1.40^{-1}$ & $1.70^{-1}$ & 0.795 & 0.694 & 0.772 & 0.806 \\
\hline & & $M_{1}$ & 1.35 & 1.18 & 0.769 & $\underline{0.674}$ & 0.761 & 0.799 \\
\hline & & $M_{1}^{*}$ & 1.35 & $1.20^{-1}$ & 0.750 & 0.687 & $\underline{0.739}$ & $\underline{0.794}$ \\
\hline & & $M_{2}$ & $1.42^{-1}$ & 1.17 & $\underline{0.746}$ & 0.690 & $\overline{0.751}$ & $\overline{0.803}$ \\
\hline & & $M_{2}^{*}$ & $1.41^{-1}$ & $1.20^{-1}$ & $\overline{0.771}$ & 0.680 & 0.753 & 0.796 \\
\hline \multirow[t]{6}{*}{1,000} & \multirow[t]{6}{*}{0.10} & $M$ & 1.31 & 1.51 & 0.750 & 0.665 & 0.772 & 0.794 \\
\hline & & $M^{*}$ & $1.35^{-1}$ & $1.55^{-1}$ & 0.762 & $\underline{0.662}$ & 0.781 & 0.802 \\
\hline & & $M_{1}$ & 1.34 & 1.09 & 0.749 & 0.687 & 0.772 & 0.804 \\
\hline & & $M_{1}^{*}$ & 1.34 & $1.30^{-1}$ & 0.714 & 0.713 & 0.768 & 0.802 \\
\hline & & $M_{2}$ & $1.39^{-1}$ & 1.22 & $\underline{0.695}$ & 0.727 & 0.759 & 0.802 \\
\hline & & $M_{2}{ }^{*}$ & $1.38^{-1}$ & $1.14^{-1}$ & 0.725 & 0.664 & $\underline{0.755}$ & $\underline{0.794}$ \\
\hline \multirow[t]{6}{*}{500} & \multirow[t]{6}{*}{0.20} & $M$ & 1.35 & 1.65 & 0.767 & 0.669 & 0.786 & 0.807 \\
\hline & & $M^{*}$ & $1.40^{-1}$ & $1.56^{-1}$ & 0.750 & $\underline{0.660}$ & 0.769 & 0.798 \\
\hline & & $M_{1}$ & 1.42 & 1.19 & 0.729 & 0.669 & $\underline{0.744}$ & $\underline{0.795}$ \\
\hline & & $M_{1}^{*}$ & 1.43 & $1.20^{-1}$ & 0.691 & 0.745 & $\overline{0.778}$ & $\overline{0.800}$ \\
\hline & & $M_{2}$ & $1.48^{-1}$ & 1.20 & $\underline{0.656}$ & 0.781 & 0.784 & 0.798 \\
\hline & & $M_{2}{ }^{*}$ & $1.45^{-1}$ & $1.20^{-1}$ & 0.725 & 0.683 & 0.745 & 0.801 \\
\hline
\end{tabular}

Sample size $N=N_{0}=N_{1}$.

The underlined value is the minimum power of a test across the 6 genetic models.

power of 0.746 and is the second-best test. On the other hand, MIN2 has a minimum power of 0.744 in the case of high-frequency SNPs and is here the second-best test. Note that since MIN2 tended to be slightly conservative using low-frequency SNPs, when asymptotic results were used for computing the $\mathrm{p}$ values, it is thus fair to say that MIN2 might have better efficiency robustness if one uses a better method to compute its $\mathrm{p}$ values.

\section{Applications}

\section{An Ovarian Cancer Association Consortium Study}

The Ovarian Cancer Association Consortium Study (OCAC) considered here consists of 3 initial case-control studies in the USA and 12 additional follow-up case-control studies that were part of the original study. In the 3
Table 7. Application to the Ovarian Cancer Association Consortium Study

\begin{tabular}{lllll}
\hline Study & MAX3 & CLRT & MIN2 & IND \\
\hline Initial 3 studies & & & & \\
$\quad$ DOV & $\mathbf{0 . 0 3 2 3}$ & 0.0967 & $\mathbf{0 . 0 2 7 7}$ & $\mathbf{0 . 0 3 1 0}$ \\
HOP & 0.9062 & 0.2564 & 0.8550 & 0.8524 \\
USC & $\mathbf{0 . 0 1 8 4}$ & $\mathbf{0 . 0 3 4 4}$ & 0.0579 & 0.0672 \\
Additional 12 studies & & & & \\
AUS & 0.1203 & 0.1654 & 0.2451 & 0.2611 \\
GER & 0.3673 & 0.3968 & 0.4865 & 0.4613 \\
HAW & 0.6079 & 0.2991 & 0.6572 & 0.6079 \\
MAL & 0.1766 & 0.1855 & 0.1739 & 0.1390 \\
MAY & 0.4441 & 0.7671 & 0.6808 & 0.6377 \\
NCO & 0.5297 & 0.7386 & 0.3921 & 0.4374 \\
NEC & 0.7938 & 0.2213 & 0.7734 & 0.6832 \\
POL & 0.1376 & 0.0618 & 0.1811 & 0.1065 \\
SEA & 0.6433 & 0.2137 & 0.5767 & 0.4878 \\
STA & 0.5380 & 0.1983 & 0.7924 & 0.7426 \\
UCI & 0.1203 & 0.1154 & 0.0885 & 0.0963 \\
UKO & 0.3776 & 0.3331 & 0.4735 & 0.4732 \\
\hline Initial 3 studies & $\mathbf{0 . 0 1 1 5}$ & $\mathbf{0 . 0 0 4 3}$ & $\mathbf{0 . 0 0 8 4}$ & $\mathbf{0 . 0 0 6 7}$ \\
\hline Additional 12 studies & $\mathbf{0 . 0 4 5 0}$ & $\mathbf{0 . 0 1 8 2}$ & 0.0707 & $\mathbf{0 . 0 2 8 5}$ \\
\hline All & $\mathbf{0 . 0 0 4 7}$ & $\mathbf{0 . 0 0 4 8}$ & $\mathbf{0 . 0 0 4 0}$ & $\mathbf{0 . 0 0 3 0}$ \\
\hline
\end{tabular}

The values are p values. For the study abbreviations, see Doherty et al. [20].

Bold values indicate that the association tests are significant at the $5 \%$ level.

initial studies, the analyses were restricted to 3,315 nonHispanic white women who were successfully genotyped for SNP rs 2295190 located $19 \mathrm{~kb}$ downstream of the ESR1 gene. The sample included 1,449 cases and 1,866 controls. In the 12 additional follow-up case-control studies, there were 6,417 non-Hispanic white female cases and 7,450 non-Hispanic white female controls. Details on material, methods and quality control of the genotyping have been reported by Doherty et al. [20]. Here, we focus on detecting the association signals based on the 15 individual and 3 combined studies. We applied the 4 association tests considered in this paper to these studies; their $\mathrm{p}$ values are presented in table 7. The p values for the MAX3 and IND tests were computed based on using 10,000 permutation steps.

Most importantly, we note that, using a $5 \%$ significance level, no association signals can be detected from any individual study, except the studies at sites DOV and USC, where only marginal signals were detected. However, for the combined studies, all tests were able to show strong evidence that rs2295190 was associated with an 
Table 8. Application to a prostate cancer study

\begin{tabular}{|c|c|c|c|c|c|c|c|c|}
\hline \multirow[t]{2}{*}{ Population } & \multirow[t]{2}{*}{ Gene } & \multirow[t]{2}{*}{ SNP } & \multicolumn{2}{|l|}{ MAF } & \multirow[t]{2}{*}{ MAX3 } & \multirow[t]{2}{*}{ CLRT } & \multirow[t]{2}{*}{ MIN2 } & \multirow[t]{2}{*}{ IND } \\
\hline & & & in cases & in controls & & & & \\
\hline \multirow[t]{4}{*}{ Caucasians } & MSR1 & rs12718376 & 0.107 & 0.153 & 0.0054 & 0.0097 & 0.0039 & 0.0040 \\
\hline & MSR1 & rs351572 & 0.438 & 0.404 & 0.0153 & 0.0124 & 0.0238 & 0.0086 \\
\hline & ELAC2 & rs17552022 & 0.122 & 0.091 & 0.0339 & 0.0514 & 0.0287 & 0.0289 \\
\hline & ELAC2* & rs11545302 & 0.268 & 0.189 & $3.1 \mathrm{E}-5$ & 2.49E-5 & $1.79 \mathrm{E}-5$ & $2.10 \mathrm{E}-5$ \\
\hline \multirow[t]{4}{*}{ Hispanics } & RNASEL & rs627928 & 0.513 & 0.467 & 0.1482 & 0.1530 & 0.2513 & 0.5134 \\
\hline & RNASEL & rs486907 & 0.313 & 0.244 & 0.0032 & 0.0057 & 0.0109 & 0.0177 \\
\hline & RNASEL & rs682585 & 0.446 & 0.486 & 0.0673 & 0.0328 & 0.1002 & 0.0399 \\
\hline & MSR1 & rs12114368 & 0.169 & 0.208 & 0.0658 & 0.0251 & 0.0653 & 0.0364 \\
\hline \multirow[t]{2}{*}{ African Americans } & MSR1 & rs4333601 & 0.433 & 0.540 & 0.0496 & 0.0225 & 0.0353 & 0.0438 \\
\hline & MSR1 & rs351572 & 0.341 & 0.256 & 0.0612 & 0.0734 & 0.0640 & 0.0367 \\
\hline
\end{tabular}

The values are $\mathrm{p}$ values. Bold values indicate that the association tests are significant at the $5 \%$ level.

increased invasive ovarian cancer risk. When all 15 individual studies were combined into a single analysis, we found that the IND test showed the strongest association signal $(\mathrm{p}=0.0030)$. From all analyses, we also found that the MIN2 and IND tests seem to have similar $p$ values.

\section{A Prostate Cancer Study}

The prostate cancer study considered here originally included men from the San Antonio Center for Biomarkers of Risk of Prostate Cancer cohort. From this cohort, 226 incident cases were available. Additionally, 646 cases with a known history of prostate cancer were included who were enrolled within the same time period in a parallel study of prevalent prostate cancer using the same recruiting strategies. Male volunteers ( $\geq 45$ years old) served as controls. They had normal digital rectal examinations and prostate-specific antigen levels $<2.5 \mathrm{ng} / \mathrm{ml}$ on all study visits. Ethnicity was self-reported in a questionnaire completed at the time of enrollment. A total of 1,436 Caucasians (596 cases, 840 controls), 648 Hispanics (194 cases, 454 controls) and 270 African Americans (82 cases, 188 controls) were included in the analysis. For each subject, 39 SNPs were genotyped. Details of the SNP selection and genotyping procedure have been reported by Beuten et al. [21]. Here, we selected some important SNPs for demonstration. To avoid possible population stratification, association analyses were done separately for each ethic group. The $\mathrm{p}$ values of the 4 association tests are reported in table 8 .

As can be seen in table 8 , except in some cases, the IND test tends to have smaller $\mathrm{p}$ values when there was a stronger association between SNPs and the prostate cancer risk. Using a 5\% significance level, 4 SNPs could not be detected if the MAX3 or MIN2 tests were used. On the other hand, 3 SNPs could not be detected by the CLRT test. Only 1 SNP could not be detected by the IND test.

\section{Discussion}

There are many tests available for detecting genetic association signals in case-control studies. Since the genetic models of the SNPs are usually unknown, it is preferable to use robust tests $[7,9,10,22]$. When the genetic models were limited to the DOM, ADD, MUL and REC models and the MAFs were restricted to those $\geq 0.10$, Joo et al. [7] found that the MAX3 and CLRT tests had greater efficiency robustness. When other genetic models (such as the overdominant models) were also included for comparison, they found that both Pearson's $\chi_{2}^{2}$ and the MIN2 test had greater efficiency robustness. However, our unreported simulation results indicated that, in cases with a low MAF, both Pearson's $\chi_{2}^{2}$ test and the 2-df likelihood ratio test based on logistic regression had low efficiency. The CATT-type tests had low efficiency robustness in cases with a low or high MAF. Recently, Chen and $\mathrm{Ng}$ [11] considered a generalized order-restricted relative-risk model and showed that their test was more robust than the CATT-type tests. Unfortunately, this and other conditions mentioned in the literature are difficult to verify in real applications.
44 
Here, we first pointed out that the $2 \times 2$ tables constructed for this study might carry different association signals under different SNPs and genetic models. We showed that, by properly choosing a table, one can develop a test which is not only efficient but also more robust, so that the impact of misspecifying the genetic model and allele frequency is small. The suggested test statistic is a sum of Pearson's $\chi^{2}$ statistics for independence testing for 3 selected $2 \times 2$ tables. This statistic is closely related to the sequence kernel association test and sum of squared score test statistics. They only differ in using different weights for Pearson's $\chi^{2}$ statistics. In this paper, we have done extensive simulations to investigate the properties of the new test. We reported the simulated properties of the 4 competing tests when the case and control data were balanced. The simulated results indicated that the new test was almost uniformly most powerful across a wide spectrum of genetic models. Unreported simulated results, when cases and controls were imbalanced, also showed that the new test was relatively more efficient and robust. In the simulations, the 2 - $\mathrm{df}$ likelihood ratio test under the logistic model was also studied. Unreported simulation results indicated that the likelihood ratio test was also less powerful than the IND test and that its power was more sensitive to allele frequency.

It is also of interest to investigate the properties of the IND test in genome-wide association studies, in which one often needs to test 100,000-500,000 SNPs simultaneously. Unfortunately, the direct computation of the $p$ values for the IND test across all SNPs is intensive if the permutation method is used. For example, in the simulations, if we used a more stringent significance level of 0.0001 and 100,000 replications, it would take us about 10 days to complete the study. To solve the computation problem, the asymptotic distribution of the IND test may be used to compute the $\mathrm{p}$ values. The limited simulation results given in this paper have shown that the permutation-based and asymptotic-based IND tests have a very similar performance in terms of type I error rate and power, particularly in the case of high-frequency SNPs. However, the computation time of the asymptotic-based IND test was substantially reduced.

Note that the IND test can be extended to include covariates. One general approach is to consider IND-type statistics based on the $D \times G$ tables conditional on each covariate level. The overall test statistic can then be defined as the sum of these IND-type statistics. The asymptotic distribution of the test statistic is equivalent to the distribution of a weighted sum of correlated $\chi^{2}$ random variables. However, accounting for a covariate is not al- ways necessary. For example, under the logistic regression model, the IND test based on the marginal $D \times G$ table is still valid even when the covariate and the genetic factor are independent and there is no SNP-covariate interaction. However, the test may be less powerful, depending on the distribution of the covariate(s).

Our proposed test can also easily be generalized for testing the presence of the main and/or SNP-SNP interaction effects. Suppose there are 2 SNPs, $G_{1}^{*}$ and $G_{2}^{*}$, where the former is the major SNP and the latter is secondary, and one wishes to test whether $G_{1}^{*}$ has main and/ or $G_{1}^{*} \times G_{2}^{*}$ interaction effects. If $G_{1}^{*}$ and $G_{2}^{*}$ are dependent, we may define similar IND statistics by treating $G_{2}^{*}$ as a covariate. On the other hand, if $G_{1}^{*}$ and $G_{2}^{*}$ are independent in the control population (e.g. the 2 SNPs are from different genetic regions), then the interaction effects can be more efficiently assessed by using the caseonly data; see Cheng [23]. In this situation, we define 2 statistics: one for detecting a $D-G_{1}^{*}$ association signal, the other one for detecting a $G_{1}^{*}-G_{2}^{*}$ association signal based on using only the case data. The sum of these 2 IND-type statistics can be used for testing whether $G_{1}^{*}$ has main and/or interaction effects. We are currently investigating the properties of these methods and the results will be reported elsewhere.

\section{Appendix}

\section{Asymptotic Distribution of the IND Test}

To derive the asymptotic distribution of the IND test, it is more convenient to express the test statistic in terms of data in the $2 \times 3$ table. Let $N_{0, i}$ denote the frequency of observing $G=i$ in the control sample with size $N_{0}$. The corresponding terms in the case sample are denoted by $N_{1, i}$ and $N_{1}$, respectively. Using the given notation, our test statistic can be written as

$$
\mathrm{IND}=\sum_{i=1}^{3} c_{i} Z_{i}^{2},
$$

where for $i=1$ and $2, c_{i}=\left(N_{0}+N_{1}-N_{+, 3-i}\right) /\left(N_{1}-N_{1,3-i}\right)$ $\left(N_{0}-N_{0,3-i}\right) N_{+, 0} N_{+, i}$ and $N_{+, i}=N_{0, i}+N_{1, i}$, and $c_{3}=\left(N_{0}+N_{1}\right) /$ $N_{1} N_{0} N_{+, 0}\left(N_{1}+N_{0}-N_{+, 0}\right)$. Further, $Z_{i}=N_{1,0} N_{0, i}-N_{1, i} N_{0,0}$, where $N_{0,3}=N_{0,1}+N_{0,2}$ and $N_{1,3}=N_{1,1}+N_{1,2}$. The asymptotic distribution of the IND test can be expressed in terms of the asymptotic distribution of the random vector $\left(Z_{1}, Z_{2}, Z_{3}\right)$. Since it is asymptotic, $\left(Z_{1}, Z_{2}, Z_{3}\right)$ has a zero mean multivariate normal distribution, therefore the IND test is asymptotically equivalent to a weighted sum of correlated $\chi^{2}$ random variables. The distribution of the IND test can be approximated by the simulated distribution of

$$
\sum_{i=1}^{3} c_{i} Z_{i}^{2}
$$

based on the simulated data generated from the multivariate normal distribution, if the variance-covariance matrix of the distribution is known. 
Note that under the null hypothesis, the variance-covariance matrix of $\left(Z_{1}, Z_{2}, Z_{3}\right)$ is given by

$$
\operatorname{Var}_{i}=N_{0} N_{1} P_{0} P_{i}\left\{2+\left(N_{0}+N_{1}-2\right)\left(P_{0}+P_{i}\right)\right\},
$$

for $i=1,2,3$, where $P_{i}=\operatorname{Pr}(G=i)$ for $i=0,1,2$, and $P_{3}=P_{1}+P_{2}$. Further,

$$
\operatorname{COV}\left(Z_{1}, Z_{2}\right)=N_{0} N_{1}\left(N_{0}+N_{1}-2\right) P_{0} P_{1} P_{2}
$$

and

$$
\begin{aligned}
& \operatorname{COV}\left(Z_{i}, Z_{3}\right)=N_{0} N_{1}\left(N_{0}+N_{1}-2\right) P_{0}\left(P_{i}^{2}+P_{0} P_{i}+P_{1} P_{2}\right)+ \\
& 2 N_{0} N_{1} P_{0} P_{1},
\end{aligned}
$$

for $i=1,2$. In practical applications, the unknown quantities $P_{i}$ can be replaced by their sample proportions based on the combined case and control sample.

\section{Acknowledgements}

The research was supported in part by the National Science Council (grant No. 100-2118-M-039-0010MY3).The authors are very grateful to the two reviewers for their constructive comments, which have improved the presentation of this paper.

\section{References}

$>1$ Sasieni PD: From genotypes to genes: doubling the sample size. Biometrics 1997;53: 1253-1261.

-2 Zheng G, Freidlin B, Li Z, Gastwirth JL: Choice of scores in trend tests for case-control studies of candidate-gene associations. Biom J 2003;45:335-348.

$>3$ Lettre G, Lange C, Hirschhorn JN: Genetic model testing and statistical power in population-based association studies of quantitative traits. Genet Epidemiol 2007;31:358-362.

-4 Freidlin B, Zheng G, Li Z, Gastwirth JL: Trend tests for case-control studies of genetic markers: power, sample size and robustness. Hum Hered 2002;53:146-152.

5 Balding D: A tutorial on statistical methods for population association studies. Nat Res Genet 2006;7:781-791.

6 The Wellcome Trust Case Control Consortium (WTCCC): Genome-wide association study of 14,000 cases of seven common diseases and 3,000 shared controls. Nature 2007; 447:661-678.

$>7$ Joo J, Kwak M, Ahn K, Zheng G: A robust genome-wide statistic of the Wellcome Trust Case-Control Consortium. Biometrics 2009; 65:1115-1122.

8 Wang K, Sheffield VC: A constrained-likelihood approach to marker-trait association studies. Am J Hum Genet 2005;77:768-780.
-9 Conneely KN, Boehnke M: So many correlated tests, so little time! Rapid adjustment of $\mathrm{P}$ values for multiple correlated tests. Am J Hum Genet 2007;81:1158-1168.

10 Li Q, Zheng G, Li Z, Yu K: Efficient approximation of $\mathrm{P}$-value of the maximum of correlated tests, with applications to genome-wide association studies. Ann Hum Genet 2008;72: 397-406.

-11 Chen Z, Ng HKT: A robust method for testing association in genome-wide association studies. Hum Hered 2012;73:26-34.

12 Asimit J, Zeggini E: Rare variant association analysis methods for complex traits. Ann Rev Genet 2010;44:293-308.

13 Bansal V, Libiger O, Torkamani A, Schork NJ: Statistical analysis strategies for association studies involving rare variants. Nat Rev Genet 2010;11:773-785.

14 Li B, Leal SM: Methods for detecting associations with rare variants for common disease: application to analysis of sequence data. Am J Hum Genet 2008;83:311-321.

15 Lee S, Wu MC, Lin X: Optimal tests for rare variant effects in sequencing association studies. Biostatistics 2012;13:762-775.

16 Pan W: Relationship between genomic distance-based regression and kernel machine regression for multi-marker association testing. Genet Epidemiol 2011;35:211-216.
17 Wu MC, Lee S, Cai T, Li Y, Boehnke M, Lin $\mathrm{X}$ : Rare-variant association testing for sequencing data with the sequence kernel association test. Am J Hum Genet 2011;89:82-93.

18 Lin DY, Tang ZZ: A general framework for detecting disease associations with rare variants in sequencing studies. Am J Hum Genet 2011;89:354-367.

19 Gastwirth JL: The use of maximum efficiency robust tests in combing contingency tables and survival analysis. J Am Stat Assoc 1985; 80:380-384.

20 Doherty JN, Rossing MN, Cushing-Haugen KL, et al: ESR1/SYNE1 polymorphism and invasive epithelial ovarian cancer risk: an Ovarian Cancer Association Consortium Study. Cancer Epidemiol Biomarkers Prev 2010;19: 245-250.

21 Beuten J, Gelfond JAL, Franke JL, Shook S, Johnson-Pais TL, Thompson IM, Leach RJ: Single and multivariate associations of MSR1, ELAC2, and RNASEL with prostate cancer in an ethnic diverse cohort of men. Cancer Epidemiol Biomarkers Prev 2010;19:588-599.

22 Gonzalez JR, Carrasco JL, Dudbridge F, Armengol L, Estiville X, Moreno V: Maximizing association statistics over genetic models. Genet Epidemiol 2008;32:246-254.

23 Cheng KF: A maximum likelihood method for studying gene-environment interactions under conditional independence of genotype and exposure. Stat Med 2006;25:3093-3109. 